\title{
Uso de técnicas de impresión 3D en la reconstrucción mandibular. Una revisión breve
}

Use of 3D printing techniques in mandibular reconstruction. A brief review

\section{Uso de técnicas de impressão 3D na reconstrução mandibular. Uma} breve revisão.

Nicole Escalona-Contreras ${ }^{1 凶} \underline{\mathrm{ORCID}}$, Paula Merino-Kutscher ${ }^{1 凶} \underline{\mathrm{ORCID}}$, Ricardo

Cartes-Velásquez $2 \bowtie \underline{\text { ORCID }}$

${ }^{1}$ Facultad de Odontología, Universidad Andrés Bello, Concepción, Chile.

${ }^{2}$ Fundación Kimntrum, Concepción, Chile.

Fecha correspondencia:

Recibido: marzo de 2021.

Aprobado: septiembre de 2021.

Forma de citar:

Escalona-Contreras N, Merino-

Kutscher P, Cartes-Velásquez R.

Uso de técnicas de impresión 3D

en la reconstrucción mandibular.

Una revisión breve. Rev. CES

Odont 2021; 34(2): 159-172.

https://doi.org/10.21615/

cesodon.5939

Open access

(C) Derecho de autor

Licencia creative commons

Ética de publicaciones

Revisión por pares

Gestión por Open Journal System

DOI: 10.21615/cesodon.5939

ISSNe 2215-9185

ISSN 0120-971X

Publica con nosotros

\section{Resumen}

En los últimos años, la tecnología y la medicina han podido complementarse para la optimización de tiempo, conocimientos y recursos, aumentando la posibilidad de tratamientos personalizados en más pacientes. A nivel maxilofacial, la reconstrucción de defectos mandibulares se ha visto en la necesidad de progresar sus técnicas debido a la serie de orígenes que afectan el hueso mandibular de manera drástica, como lesiones traumáticas, cáncer, infecciones, enfermedad congénita u otros, y las innumerables consecuencias tanto estéticas como funcionales, restringiendo significativamente la calidad de vida. El objetivo de este artículo es revisar conceptos básicos del uso de tecnologías de impresión 3D en la reconstrucción mandibular. La impresión 3D ha aparecido en diversos ámbitos, siendo en el área de la medicina, un aporte fundamental para la creación de formas anatómicas de alta precisión con el que se pueden diseñar objetos con reproducción de detalles de manera rápida, luego de una serie de pasos que incluyen la obtención de una imagen radiográfica, uso de software y reparación de archivos, y la obtención de un 
modelo tridimensional. Los últimos estudios han validado el uso de impresiones 3D para una reconstrucción mandibular, con claros beneficios de costos y calidad de detalles. Es necesario crear un enfoque en las técnicas quirúrgicas utilizadas con un objeto impreso tridimensionalmente $\mathrm{y}$ un análisis post operatorio de los pacientes sometidos a estos procedimientos más allá de los detalles técnicos.

Palabras clave: reconstrucción mandibular; imagenología tridimesional; oclusión dental; trasplante óseo; procedimientos quirúrgicos reconstructivos.

\section{Abstract}

In recent years, technology and medicine have been able to complement each other to optimize time, knowledge and resources, increasing the possibility of personalized treatments in more patients. At the maxillofacial level, the reconstruction of mandibular defects has seen the need to progress its techniques due to the series of origins that drastically affect the mandibular bone, such as traumatic injuries, cancer, infections, congenital disease or others, and the innumerable both aesthetic and functional consequences, significantly restricting the quality of life. The objective of this article is to review basic concepts of the use of 3D printing technologies in mandibular reconstruction. 3D printing has appeared in various fields, being in the area of medicine, a fundamental contribution to the creation of high-precision anatomical shapes with which objects with reproduction of details can be designed quickly, after a series of steps which include obtaining a radiographic image, use of software and file repair, and obtaining a threedimensional model. The latest studies have validated the use of 3D impressions for mandibular reconstruction, with clear benefits in cost and quality of details. It is necessary to create a focus on the surgical techniques used with a three-dimensional printed object and a post-operative analysis of the patients undergoing these procedures beyond the technical details.

Keywords: mandibular reconstruction; imaging three-dimensional; dental occlusion; bone transplantation; reconstructive surgical procedures.

\section{Resumo}

Nos últimos anos, tecnologia e medicina têm se complementado para otimizar tempo, conhecimento e recursos, aumentando a possibilidade de tratamentos personalizados em mais pacientes. No nível maxilofacial, a reconstrução dos defeitos mandibulares tem visto a necessidade de progredir em suas técnicas devido à série de origens que afetam drasticamente o osso mandibular, como lesões traumáticas, câncer, infecções, doenças congênitas ou outras, e as inúmeras ambas estéticas. e consequências funcionais, restringindo significativamente a 
qualidade de vida. O objetivo deste artigo é revisar os conceitos básicos do uso das tecnologias de impressão 3D na reconstrução mandibular. A impressão 3D tem surgido em vários campos, sendo na área da medicina uma contribuição fundamental para a criação de formas anatômicas de alta precisão com as quais objetos com reprodução de detalhes podem ser desenhados rapidamente, após uma série de etapas que incluem a obtenção de uma imagem radiográfica , uso de software e reparo de arquivos e obtenção de um modelo tridimensional. Os estudos mais recentes têm validado o uso de impressões 3D para reconstruções mandibulares, com claros benefícios em custo e qualidade de detalhes. É necessário criar um foco nas técnicas cirúrgicas utilizadas com um objeto impresso tridimensional e uma análise pós-operatória dos pacientes submetidos a esses procedimentos para além dos detalhes técnicos.

Palavras-chave: reconstrução mandibular; imagem tridimensional; oclusão dentária; transplante ósseo; procedimentos cirúrgicos reconstrutivos.

\section{Introducción}

La impresión 3D comenzó en los años 70 al mismo tiempo que Charles W. Hull inventó un método de estereolitografía (SLA) que se utilizó para la producción de maquetas; este método es el prólogo para la creación de impresoras 3D ${ }^{(1)}$. Este mecanismo ha integrado numerosos mercados a medida que las impresoras progresan desde la construcción de piezas simples y prototipos hasta la creación de componentes y productos finales completamente funcionales, como baterías ${ }^{(2)}$.

La planificación y simulación quirúrgica asistida por computadora ahora se utilizan regularmente para el análisis de estructuras craneofaciales ${ }^{(3)}$. Desde su introducción en el campo de la medicina a fines de la década de 1980, las impresoras 3D han construido una gama de dispositivos, como prótesis dentales, audífonos y prótesis de cadera ${ }^{(3)}$. Esta tecnología aumenta las posibilidades de tratamiento personalizado para los pacientes, al tiempo que reduce los costos de fabricación ${ }^{(4)}$.

En los últimos años, el desarrollo de la tecnología de impresión 3D para la ingeniería de tejidos ha contribuido a aumentar la velocidad y la precisión desde la planificación hasta la operación en la reconstrucción maxilofacial ${ }^{(5)}$. La tecnología 3D ha sido investigada en la fabricación de andamios con factores de crecimiento junto con una guía y plantilla quirúrgica. En el último 
tiempo ha sido capaz no solo de reproducir la complejidad anatómica individual de un defecto maxilofacial, sino también de optimizar funciones y propiedades mecánicas mediante la combinación de diversos materiales, células madre y factores de crecimiento ${ }^{(5)}$.

El objetivo de este artículo es revisar conceptos básicos del uso de tecnologías de impresión 3D en la reconstrucción mandibular.

\section{Reconstrucción Mandibular}

Múltiples procesos de la enfermedad, incluyendo neoplasia, trauma y efectos secundarios de medicamentos, requieren resección segmentaria y posterior reconstrucción de la mandíbula. La pérdida de continuidad mandibular puede causar una variedad de problemas que incluyen compromiso de las vías respiratorias, dificultad con la masticación, debido a la pérdida de piezas dentarias o maloclusión, disartria, disfagia, y falta de estética debido a la pérdida del contorno facial inferior ${ }^{(6)}$. Puede haber defectos de tejidos blandos asociados a estructuras adyacentes que involucran, por ejemplo, la lengua, la mucosa bucal, cuello y / o la piel de la cara, que pueden tener consecuencias funcionales y estéticas igualmente importantes ${ }^{(6)}$.

A medida que las técnicas quirúrgicas han avanzado, se han desarrollado varias tecnologías con el potencial de transformar significativamente el enfoque de los cirujanos en materias de restauración de la continuidad mandibular (7). Los defectos mandibulares grandes son clínicamente difíciles de reconstruir, debido a la compleja anatomía de la mandíbula y la disponibilidad limitada de tejido apropiado para la reparación ${ }^{(8)}$. (Figuras $\underline{1}$ y $\underline{2}$ ).

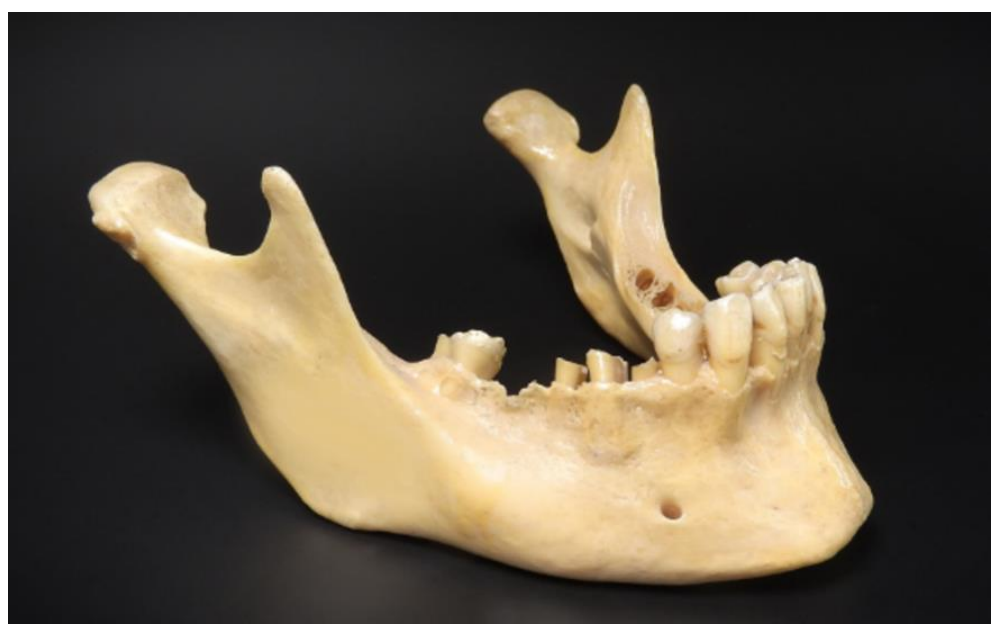

Figura 1. Vista mediolateral de mandíbula.

De MAKY.OREL tiene licencia de CC 1.0. 


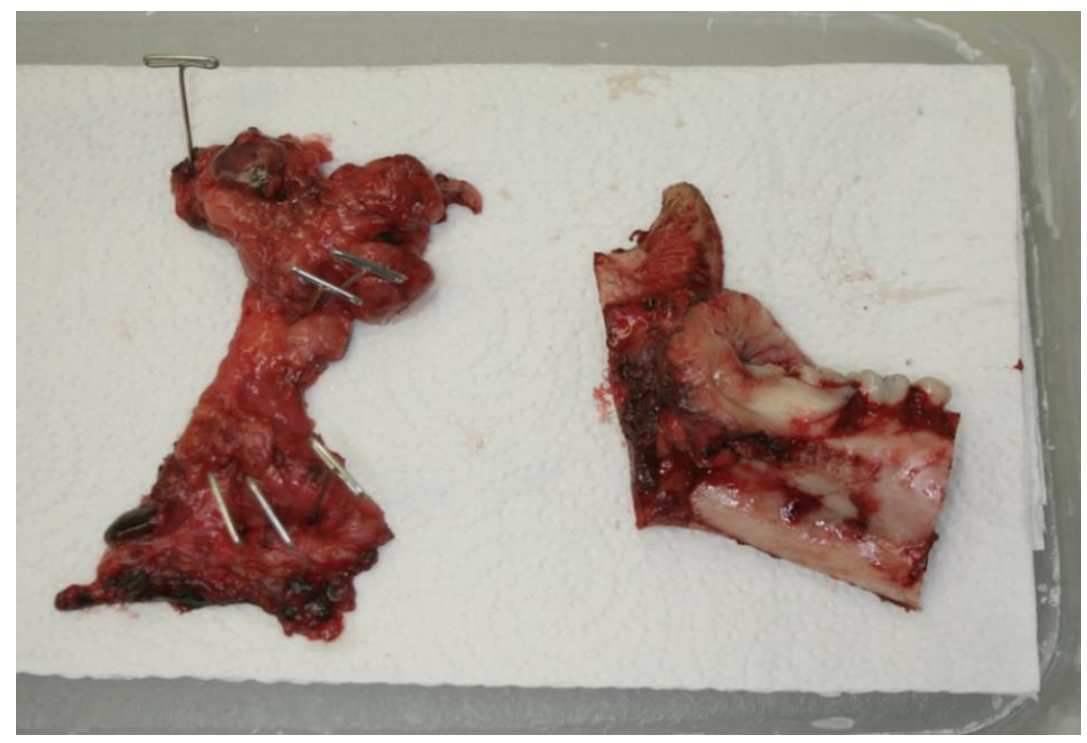

Figura 2. Disección mandibular por cáncer oral. La imagen tiene licencia por Hapshaw CC BY-NC-SA 2.0.

Desde un punto de vista funcional, psicológico y socioeconómico, los defectos mandibulares grandes tienen efectos devastadores en la calidad de vida. Restaurar tanto la función mandibular como la estructura estética es clave para un resultado exitoso ${ }^{(8)}$. El gold standard actual para la reconstrucción mandibular es el colgajo libre de peroné, en el que se extrae un segmento de este, se da forma manualmente al tamaño y geometría del defecto mandibular en el pabellón quirúrgico, y se transfiere como un colgajo libre de hueso a la mandíbula ${ }^{(9)}$.

La búsqueda del método ideal para reparar defectos óseos ha sido objeto de esfuerzos a largo plazo de distintas disciplinas relacionadas. Si bien, el injerto autólogo tradicional es el gold standard $^{(10)}$, la escasez de fuentes y las complicaciones de los donantes (como el dolor crónico y la hipofunción) limitan su aplicación y son propensas a la reabsorción ósea secundaria ${ }^{(11)}$. Se espera que varios biomateriales, en combinación con nuevas tecnologías como la navegación digital y la impresión en 3D, puedan ajustarse para construir nuevos tipos de andamios con una estructura y componentes más precisos, que aborden las necesidades de la cirugía y la reconstrucción posterior ${ }^{(12)}$.

Es por ello que, en los últimos años, con el rápido desarrollo de materiales biológicos y la necesidad de la medicina regenerativa moderna, la investigación ha permitido la elaboración de biomateriales sustitutos que se han aplicado a la zona ósea mandibular ${ }^{(11)}$. Los biomateriales 
no solo tienen o cumplen ciertas funciones biológicas, sino que también tienen buena biocompatibilidad. Sin embargo, hay muchos tipos de biomateriales aplicados en defectos de la mandíbula y las propiedades pueden variar ${ }^{(11)}$.

Con el rápido desarrollo de la tecnología informática y la imagenología, la tecnología del sistema de navegación asistida por computadora (CANS) se ha aplicado ampliamente en la reconstrucción quirúrgica del tejido duro ${ }^{(11)}$. La tecnología de diseño y fabricación asistida por computadora (CAD/CAM) combinada con el trasplante de hueso y la implantación individualizada de titanio ha sido cada vez más fructífera y ha logrado resultados ideales ${ }^{(11)}$. Es por lo anteriormente mencionado con respecto a los riesgos que conlleva y los diferentes tipos de tratamiento, que se hace necesario desarrollar aún más la utilización de la tecnología de impresión 3D en la reconstrucción mandibular.

\section{Aspectos técnicos de impresiones 3D}

La impresión 3D para su utilización requiere de un equipo y software especial donde a partir de un diseño o imagen se crea un modelo tridimensional que la impresora reconoce y "lee", y a partir del cual el objeto se va construyendo por la adición "capa por capa” del material deseado (filamentos de polímeros o metales) ${ }^{(12)}$. Esta obtención de un objeto 3D, corresponde a la técnica que incluye distintas tecnologías de obtención de estructuras desde un "diseño asistido por computador" (CAD), en pocas horas y con poca intervención humana. El resultado, será una representación sólida en $3 \mathrm{D}$ del diseño desarrollado, que puede realizarse de forma manual o automática a partir de un software especializado y así obtener la estructura virtual deseada, pero a menudo se requiere una combinación entre segmentación automatizada y manual debido a la complejidad de las regiones anatómicas, y de la presencia de artefactos que modifican el umbral de intensidad ${ }^{(13)}$. Cada método de impresión ofrece sus propias ventajas y desventajas, como el tiempo de construcción, los materiales utilizados, la precisión y la durabilidad. Actualmente no existe un proceso de impresión estándar para la atención al paciente y la investigación médica ${ }^{(2)}$.

Los pasos para la elaboración de objetos mediante impresión 3D, comienza a partir de un archivo cuya base estructural es un modelo tridimensional virtual viable. En el caso de su aplicación en medicina, son requeridos estudios de imagen de un paciente para crear un molde a la medida. Esta es la representación digital de lo que se planea imprimir mediante algún programa computarizado ${ }^{(12)}$, como aislar una vista de la mandíbula, obtenida de la TC del paciente en varios planos (coronal, axial y sagital). La información obtenida mediante estos recursos se almacena en un archivo de imágenes digitales (archivo DICOM) ${ }^{(2)}$. La imagen digital 
y la comunicación en medicina se pueden traducir en archivos de estereolitografía (STL), correspondiente a un archivo informático de diseño asistido por computadora (CAD), se utiliza este archivo para optimizar, ya sea, corrección de imperfecciones o creación de un soporte, antes de la impresión y que por último son leídos y construidos metódicamente por impresoras $3 D^{(4)}$.

La traducción de DICOM a un formato legible para la impresora 3D incluye varios pasos, y cada paso puede requerir su propio software ${ }^{(2)}$, quien corrige en parte los artefactos residuales, como eliminación de partes del maxilar que quedan en la imagen, relleno de ciertos defectos internos de la mandíbula, entre otros ${ }^{(4)}$.

Existen distintos tipos de obtención mecánica de un modelado rápido, dependiendo del tipo de material que se utilice, que se pueden clasificar en técnicas de adición de capas, de sustracción y de deformación de material. Dentro de las técnicas de adición de capas encontramos la técnica de modelado por deposición fundida (FDM) ${ }^{(13)}$, donde se utilizan materiales termoplásticos, que como su nombre lo indica, adquieren la forma semilíquida a temperaturas mayores a los $120^{\circ} \mathrm{C}$, convirtiéndolo en un material fácilmente aplicable mediante una boquilla extrusora que va depositando finas capas sobre una plataforma hasta la construcción del modelo, la que se endurece a medida que la temperatura del material disminuye (12). Esta técnica permite utilizar materiales termoplásticos como el PLA (ácido poliláctico), ABS (acrilonitrilo butadieno estireno), HDPE (polietileno de alta densidad), metales sintéticos, e inclusive materiales comestibles o fármacos ${ }^{(12)}$. El modelado por deposición fundida es muy adecuado para la fabricación de réplicas de hueso, ya que utiliza materiales que elaboran prototipos duros y robustos, así como también reproduce estructuras de forma compleja como guías y modelos quirúrgicos ${ }^{(14)}$.

Con respecto a la exactitud dimensional de los biomodelos, ésta se debe evaluar antes de decidir la aplicabilidad de la estructura, pudiendo producirse errores dimensionales en las distintas etapas de la fabricación del prototipo, por lo que en la literatura se describe una exactitud dimensional aceptable, por lo menos clínicamente, entre 0,6 y el $6 \%$ en el modelado por adición de capas ${ }^{(13)}$. Esto puede aplicarse para la mayoría de los usos del prototipado rápido, pero es de crucial importancia para modelos cráneo-maxilofaciales con los que se requiere, una aún más alta precisión cuando estos son utilizados para la fabricación de guías de implantes ${ }^{(13)}$. (Figura 3). 


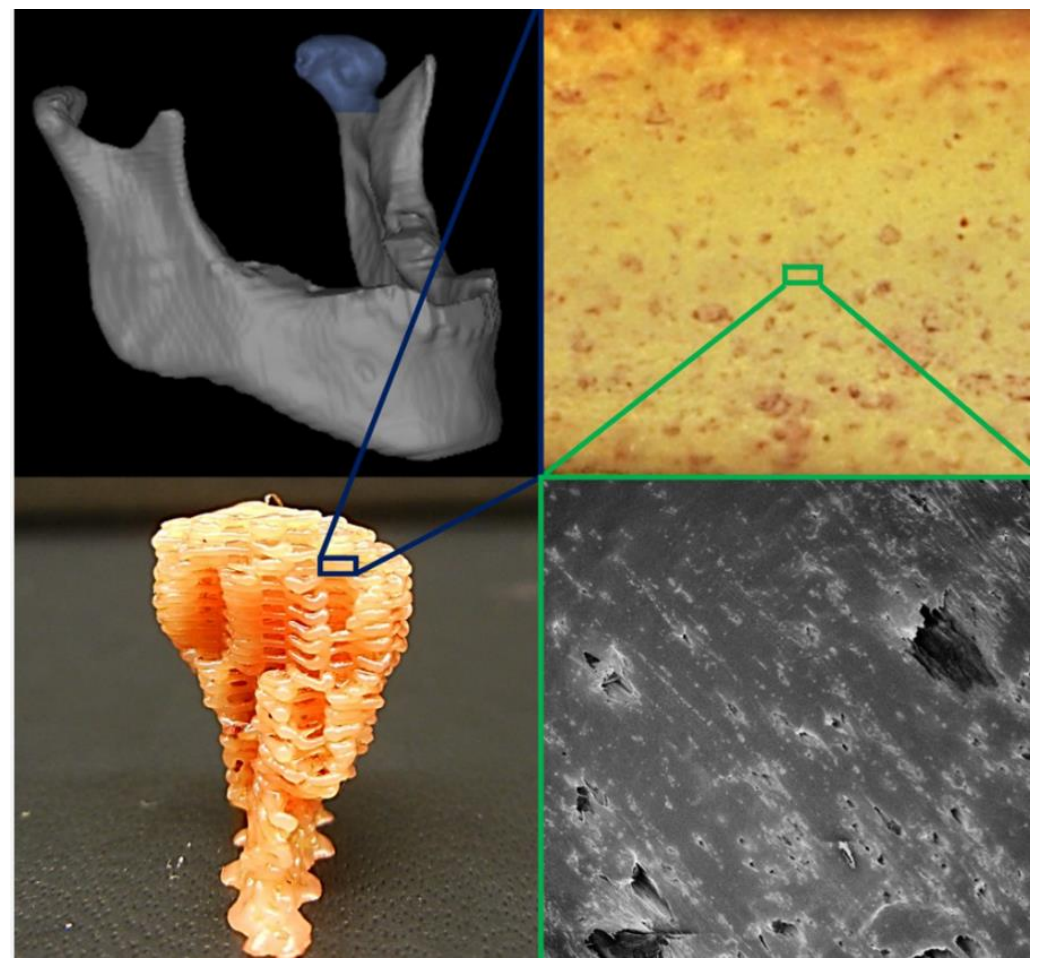

Figura 3. Impresión tridimensional de hueso en forma anatómica De los Institutos Nacionales de Salud (NIH) está autorizada bajo CC BY-NC 2.0.

Por otra parte, los métodos de visualización digital en 3D de alta precisión están ampliamente disponibles en el mercado actual. Sin embargo, esta tecnología aún requiere que los cirujanos hayan obtenido conocimientos sustanciales para interactuar autónomamente con los datos de imágenes. Varios servicios de impresión se han hecho cargo de los aspectos técnicos y proporcionan al cirujano modelos anatómicos fabricados para pacientes específicos ${ }^{(15)}$.

\section{Aspectos Clínicos de impresiones 3D (imágenes)}

Existen cuatro tipos generales de aplicaciones quirúrgicas de impresión en 3D que se pueden usar en cirugía cráneo-maxilofacial ${ }^{(16)}$ :

a. Modelos de contorno (modelos de espacio positivo para permitir la aplicación y ensayo de aditamentos antes de la cirugía),

b. Guías (modelos de espacio negativo de datos reales del paciente para guiar el corte y perforación),

c. Férulas (modelos de espacio negativo de posiciones postoperatorias virtuales para guiar la alineación final), e 
d. Implantes (materiales implantables impresos en 3D de espacio negativo o moldes impresos en 3D).

Los modelos de contorno, son la forma más común de tecnología de impresión tridimensional utilizada en la cirugía craneomaxilofacial ${ }^{(16)}$. Los modelos de contorno representan la anatomía específica del paciente que se imprime en 3D y se utiliza como una réplica exacta de la anatomía ósea del paciente, para contornear hardware como plantillas de titanio, que es más fácil en un modelo que estar intraoperatoriamente, donde las estructuras están obstruidas por tejido blando y vasos sanguíneos ${ }^{(16)}$. Este uso se evaluó en un estudio que incluyó gatos con osteosarcoma de mandíbula antes de la cirugía, y usó tecnología de procesamiento CAD / CAM para personalizar la placa de reconstrucción de titanio a través de la impresión 3D, y luego realizó una resección periódica de la mandíbula, seguida de la reconstrucción del defecto con un titanio hecho a medida en una placa intraoperatoria. Durante el seguimiento postoperatorio, no se encontraron signos de alimentación deficiente. Se encontraron cultivos normales a excepción de lesión pulpar en el área de la lesión. El gato estaba vivo y libre de enfermedad 14 meses después de la operación ${ }^{(17)}$.

Las guías quirúrgicas usan el espacio negativo que rodea la anatomía ósea de un paciente para imprimir plantillas específicas del paciente que se ajustan solo en ciertos segmentos de hueso durante la operación para guiar el corte o la perforación precisa ${ }^{(16)}$. (Figura 4).

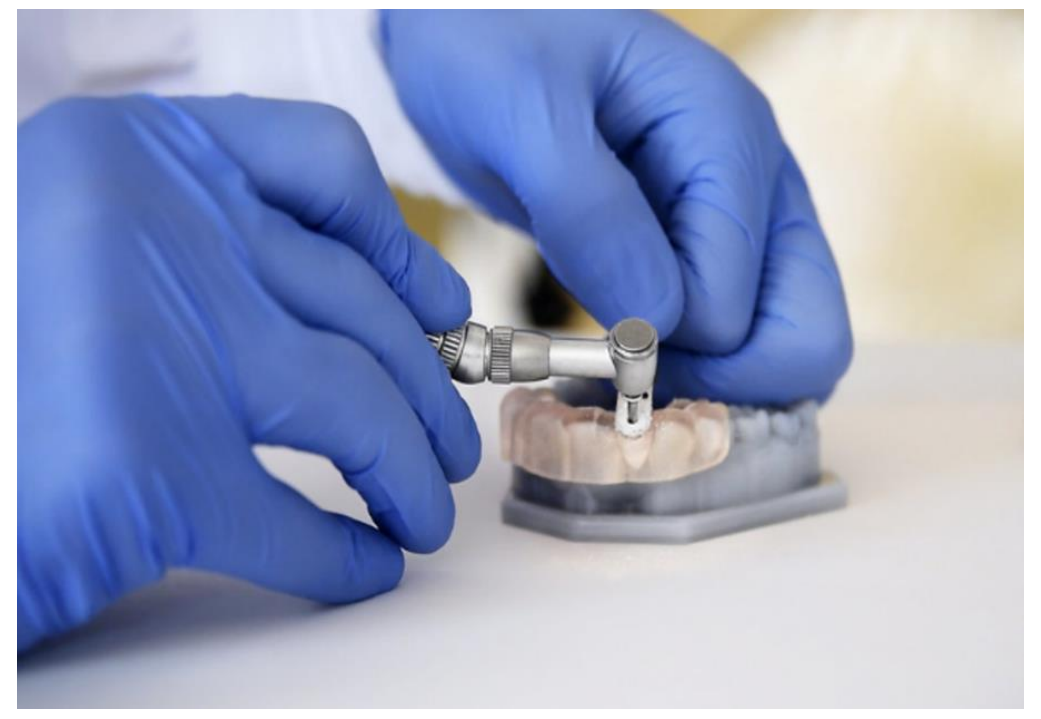

Figura 4. Guía quirúrgica impresa en 3D que ayuda a la perforación dental De formlabs Inc, tiene licencia bajo BY CC 2.0. 
Las guías son réplicas de estructuras reales de pacientes, estas se utilizan cada vez más en cirugía ortognática, reconstrucción mandibular, resecciones, injertos y reconstrucciones oncológicas ${ }^{(16)}$. Un ejemplo de este uso es un simulador digital quirúrgico (SDS, por sus siglas en inglés), el cual permite reconstrucciones más precisas y consistentes del borde inferior mandibular y del contorno facial ${ }^{(18)}$. El tiempo quirúrgico general, el tiempo de isquemia y el número de osteotomías adicionales debido a la inexactitud pueden disminuir con SDS ${ }^{(18)}$. Para este propósito, la cirugía asistida por computadora (CAS, por sus siglas en inglés) ofrece la capacidad de planificar osteotomías de los sitios de resección y de donante, reflejar la mandíbula no afectada, evaluar las relaciones de la placa ósea para el posicionamiento de los implantes dentales, crear guías de resección quirúrgica, fabricar placas de reconstrucción específicas para el paciente y, restablecer la oclusión correcta ${ }^{(19)}$. Sus ventajas son que puede simular varios esquemas quirúrgicos antes de la cirugía, llevar a cabo el diseño de la placa de guía digital, la placa de reconstrucción de titanio y la posición de fijación del implante de titanio,

optimizar la biomecánica de la prótesis (con el objetivo de proteger los ligamentos y la fijación muscular para el mantenimiento de la función de masticación y promueven la regeneración ósea), simplifican el proceso quirúrgico y acortan el tiempo de operación ${ }^{(11)}$.

Las férulas se definen como réplicas de las posiciones postoperatorias finales virtuales (inexistentes) de las estructuras del paciente. (Figura 5).

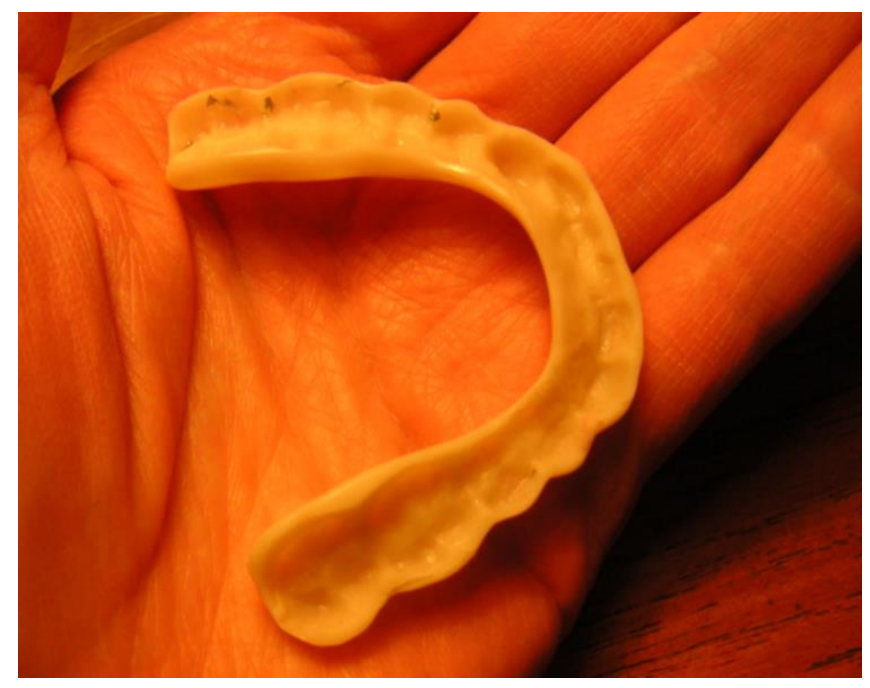

Figura 5. Guía quirúrgica impresa en 3D que ayuda a la perforación dental De formlabs Inc, tiene licencia bajo BY CC 2.0. 
Aunque las férulas también son modelos espaciales negativos, al basarse en una posición final virtual que aún no existe, esto requiere un software de diseño 3D avanzado para realizar operaciones virtuales ${ }^{(16)}$. Los beneficios que ofrece cualquier férula son: restauración de la oclusión dental pretrauma ideal, prevención de los micromovimientos del arco en los planos sagital y horizontal, reducción de la separación lingual, que no puede verificarse intraoperatoriamente, preservación de la estabilidad del arco transversal durante la fijación de los fragmentos ${ }^{(20)}$. Ejemplo de esto fue realizando ferulas con tecnologia CAD/CAM utilizando imágenes de TC del paciente, la fractura se recreó en el modelo STL generado. Los fragmentos fracturados se redujeron virtualmente a sus características anatómicas, dejando los dientes en máxima intercuspidación. Se construyó una férula virtual interoclusal correspondiente a la oclusión previa al trauma mediante una técnica aditiva ${ }^{(20)}$. Las férulas CAD / CAM generadas por la planificación virtual preoperatoria ofrecen numerosos beneficios clínicos en comparación con las férulas convencionales generadas en laboratorio: mayor precisión en la reducción de fracturas y restauración de las características anatómicas normales de la configuración del hueso fracturado en las tres dimensiones; oclusión bucal precisa y estable, así como lingual en la fase postoperatoria; excelente ajuste de la férula a la dentición durante la reducción de la fractura; mayor comodidad del paciente durante la fabricación de férulas; y fabricación y manejo fáciles, al eliminar la necesidad de procedimientos de laboratorio engorrosos ${ }^{(20)}$.

Los implantes son los objetos impresos en 3D que se implantan directamente en el paciente. (Figura 6).

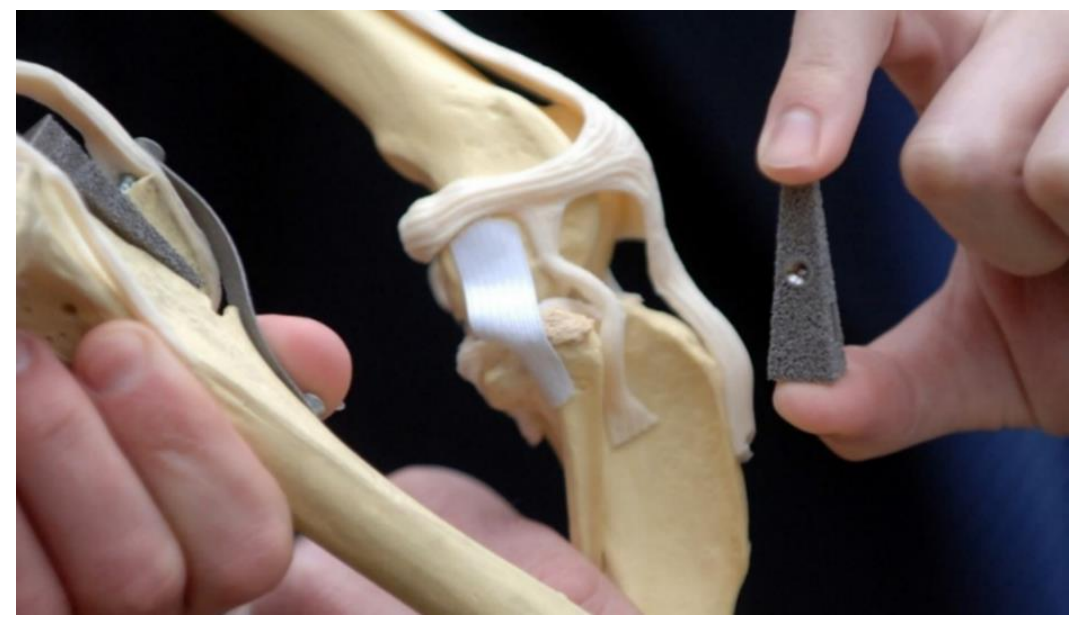

Figura 6. Implante de fusión 3dp De Andreas Kofner tiene licencia CC BY-SA 2.0. 
A veces, un objeto impreso en 3D se usa como un molde para material de implante vertible. Implantes de impresión 3D brindan soluciones estéticas a defectos óseos, y actualmente deben ser materiales biocompatibles, o usarse como andamios para tejidos diseñados ${ }^{(16)}$. Un ejemplo es el implante de biomateriales imitando las composiciones de los tejidos diana receptores ${ }^{(21)}$. Por ejemplo, en el campo de la ingeniería del tejido óseo, la matriz ósea desmineralizada (DBM) se ha utilizado ampliamente ya que reproduce el microambiente y contiene factores de crecimiento ${ }^{(22)}$. Otros usos similares incluyen la fabricación de andamios de PCL/ $\beta$-TCP y bdECM con o sin rhBMP-2, a las 12 semanas después de la cirugía, se observó calcificación y formación de novo en los grupos experimentales. La reabsorción o degradación anormal de la estructura del andamio no se observó en ningún grupo de estudio ${ }^{(5)}$.

\section{Conclusiones}

Los últimos estudios han validado el uso de impresiones 3D para una reconstrucción mandibular, con claros beneficios de costos y calidad de detalles. Es necesario crear un enfoque en las técnicas quirúrgicas utilizadas con un objeto impreso tridimensionalmente y un análisis post operatorio de los pacientes sometidos a estos procedimientos más allá de los detalles técnicos.

\section{Financiamiento}

Ninguno.

\section{Conflictos de interés}

Los autores declaran no tener conflictos de interés.

\section{Referencias}

1. Parra JC, Gallardo E, Peñaloza E. Effect of the filling percentage on tensile strength in 3D desktop printing for different printing patterns, using a randomized design of experiments. Enfoque UTE. 2019;10(4).

2. Pucci JU, Christophe BR, Sisti JA, Connolly ES. Three-dimensional printing: technologies, applications, and limitations in neurosurgery. Biotechnol Adv. 2017;35(5).

3. Lin HH, Loni $\mathrm{D}$, Lo $\mathrm{L}$. Impresión 3D en cirugía ortognática: una revisión de la literatura. JFMA. 2018;117(7):547-548. 
4. Dupret-Bories A, Vergez S, Meresse T, Brouillet F, Bertrand G. Contribución de la impresión 3D a la reconstrucción mandibular después del cáncer. EUR. 2018;135(2): 133-136.

5. Lee S, Choi D, Shim JH, Nam W. Efficacy of three-dimensionally printed polycaprolactone/beta tricalcium phosphate scaffold on mandibular reconstruction. Sci Rep. 2020;10:497.

6. Kakarala K, Shnayder Y, Tsue TT, Girod DA. Mandibular reconstruction. Oral Oncol. 2018;77:111-117.

7. Divi V. Reconstrucción Mandibular Contemporánea. Opinión Actual OtorrinolaringolCir Cabeza Cuello. 2016; 24 (5).

8. Tatara A, Koons G, Watson E. Reconstrucción mandibular asistida por biomateriales utilizando biorreactores en vivoránea. PNAS. 2019;116(14):6954-6963.

9. Hayden RE, Mullin DP, Patel AK. Reconstruction of the segmental mandibular defect: Current state of the art. Curr Opin Otolaryngol Head Neck Surg. 2012;20:231-236.

10. SarkaK K, Lee T. Regeneración de tejidos duros utilizando sustitutos óseos: una actualización sobre las innovaciones en materiales. KJIM. 2015;30(3).

11. Zhang Q, Wu W, Qian C, Xiao W, Zhu H, Guo J, et al. Biomateriales avanzados para la reparación y reconstrucción de defectos mandibular. JFMA. 2019;10.

12. Juarez $A A$, Olivos $A$, Landa $C$. Uso y aplicación de la tecnología de impresión y bioimpresión 3D en medicina. Novedades Med. 2018;61(6).

13. Salas C, Araneda L, Schott S. Evaluación del error dimensional en la reproducción tridimensional de una mandíbula humana disecada mediante prototipado rápido de modelado por deposición fundida. Int J Odontostomat. 2020;14(1).

14. Negi S, Dhiman S, Sharma RK. Basics and applications of rapid prototyping medical models. Rapid Prototyp J. 2014;20(3).

15. Hartz CR, Msallem B, Aghlmandi S, Brantner P, Thieringer FM. Can an entry-level 3D printer create high-quality anatomical models? Accuracy assessment of mandibular models printed by a desktop 3D printer and a professional device. Int J Oral Maxillofac Surg. 2020;49(1):143-148. 
16. Ghai S, Sharma Y, Jain N, Satpathy M, Pillai AK. Use of 3-D printing technologies in craniomaxillofacial surgery: a review. Oral Maxillofac Surg. 2018;22(3):249-259.

17. Zhanga Q, Wuc W, Qianb C, Xiaob W, Zhub H, Guoa J, et al. Advanced biomaterials for repairing and reconstruction of mandibular T defects. Mat Sci Eng C. 2019; 103:109858.

18. Huh JB, Bae EB, Park KH, Shim JH, Chung HY, Choi JW, et al. Efficacy of rhBMP-2 Loaded PCL/ -TCP/bdECM Scaffold Fabricated by 3D Printing Technology on Bone Regeneration. Biomed Res Int. 2018;2018:2876135.

19. Pati F, Jang J, Ha D. Impresión de análogos tisulares tridimensionales con bioenlace de matriz extracelular descelularizada. Nat Commun. 2014;5(3935).

20. Ramanathan M, Panneerselvam E, Raja KK. 3D planning in mandibular fractures using CAD/CAM surgical splints - A prospective randomized controlled clinical trial. J Craniomaxillofac Surg. 2020;48(4):405-412.

21. Groot RJ, Rieger J, Chuka R, Antoine JW, Merkx M, Speksnijder C. Functional outcomes and quality of life after segmental mandibulectomy and reconstruction with a reconstruction plate or bone graft compared to a digitally planned fibula free flap. Clinical Rsearch. 2019;32(5):393-401.

22. Van Baar G, Forouzanfar T, Liberton N, Winters H, Leusink F. Accuracy of computerassisted surgery in mandibular reconstruction: A systematic review. Oral Oncol. 2018;84(3):52-60. 